\title{
ANÁLISE E COMPARAÇÃO DE DINÂMICAS PARA TRANSFERÊNCIAS ORBITAIS NO SISTEMA TERRA-LUA
}

\section{ANALYSIS AND COMPARISON OF DYNAMICS FOR ORBITAL TRANSFERS IN THE EARTH-MOON SYSTEM}

\author{
Anderson Rodrigo Barretto Teodoro ${ }^{1}$ \\ Denilson Paulo Souza dos Santos ${ }^{2}$ \\ Antonio Fernando Bertachini de Almeida Prado ${ }^{2}$ \\ Lucas Sales Policarpo ${ }^{1}$
}

RESUMO: Neste trabalho foram analisadas manobras de transferências orbitais impulsivas com empuxo instantâneo, obtido através da transferência de Lambert, modelando uma transferência geocêntrica de um veículo deixando a órbita da Terra e chegando à órbita da Lua. O objetivo foi buscar o mínimo consumo de combustível entre as manobras, realizando uma comparação entre o método analítico, o numérico e o método probabilístico. Dessa forma, geramos gráficos, analisando e comparando os valores obtidos no método analítico e o numérico. Para o método probabilístico, foram realizados testes para avaliar a eficiência e o desempenho do algoritmo genético desenvolvido, aplicando-o na função teste de Griewank.

Palavras-chave: algoritmos genéticos; mecânica celeste; transferências orbitais.

ABSTRACT: This study analyzed impulsive orbital transfer maneuvers with impulsive thrust, based on the Lambert's problem, modeling a geocentric transfer of a vehicle leaving the Earth's orbit and achieving moon orbit. The goal was to find the minimum fuel consumption for the necessary thrust, by performing a comparison between the analytical method and the numerical probabilistic method. Thus, we generated graphs, analyzing and comparing the values obtained from the analytical and numerical method. For the probabilistic method, testing was conducted to evaluate the efficiency and performance of the genetic algorithm developed by applying the Griewank function.

Keywords: genetic algorithms; celestial mechanics; orbital transfers.

\footnotetext{
${ }^{1}$ Engenharia da Computação / ETEP Faculdades. E-mails: andersonrbteodoro@gmail.com; Isalespolicarpo@gmail.com.

2 Divisão de Mecânica e Controle / Instituto Nacional de Pesquisas Espacias - INPE. E-mails: denilson.paulo@gmail.com; prado@dem.inpe.br.
} 


\section{INTRODUÇÃO}

O estudo de manobras orbitais tem grande importância em missões espaciais, tendo diversas aplicações, como realizar transferências de órbitas em um veículo espacial e executar correções nos parâmetros orbitais de um satélite, conforme estudado em Prado (2012).

Para isso, é importante considerar durante as manobras de transferências os gastos com consumo de combustível necessário para realizar as manobras, visto que esses gastos são consideravelmente altos em uma missão espacial, e que seu fim também pode determinar o final da missão.

\subsection{Objetivos}

O objetivo do trabalho foi criar simulações computacionais através de algoritmos para comparar a aplicação de diferentes métodos para simular transferências no sistema Terra-Lua, buscando o mínimo consumo de combustível para os impulsos necessários entre as manobras de transferência dentro desse sistema.

\section{METODOLOGIAS}

Para a dinâmica do problema, aplicando o método numérico, foi utilizado o integrador Runge-Kutta de $4^{\underline{a}}$ ordem, demonstrado em Kiusalaas (2005), aplicado nas equações da dinâmica do movimento, descritas em Santos (2009). Para o método analítico, foi considerado o problema de dois corpos e foi assumido que em ambos os métodos o sistema não sofre perturbações externas. Ao final, para o método probabilístico, é aplicada uma heurística de busca e otimização através de algoritmos genéticos, conforme abordado em Linden (2008), para busca do mínimo impulso necessário para realizar a transferência entre as órbitas em ambos os métodos, buscando a otimização do consumo de combustível.

\subsection{Método de Hohmann}

Para a dinâmica do problema, aplicando o método de Hohmann, foi considerada a Terra como corpo primário e a Lua como secundário, tendo como variáveis de entrada o raio das órbitas inicial e final e obtendo como saída à magnitude do impulso e o tempo necessário para transferência entre as órbitas.

A partir dos dados fornecidos para essa simulação, foi obtido que, para realizar uma transferência de ida da Terra à Lua, são necessários, aproximadamente, 4 dias e 20 horas e uma magnitude de impulso de, aproximadamente, $3,74 \mathrm{Km} / \mathrm{s}$.

\subsection{Transferência de Lambert}

A transferência de Lambert foi realizada a partir de duas órbitas, uma inicial e outra final, onde, a partir da definição do ponto inicial da primeira órbita, é realizada a propagação da órbita para que seja feita a transferência até a órbita final desejada, conforme demonstrado na Figura 1. 


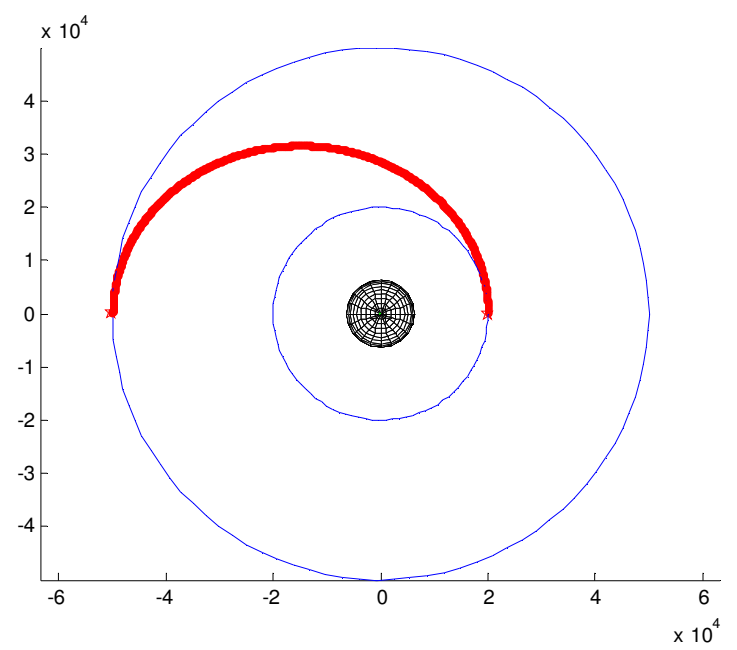

Figura 1 - Transferência de Lambert a partir de um ponto inicial até a órbita final desejada.

A partir do raio inicial, do raio final e do tempo de transferência, podemos obter o ângulo da órbita de transferência e as magnitudes dos impulsos necessários para tirar o veículo espacial da órbita inicial e colocá-lo na órbita final, conforme estudado em Curtis (2010).

\section{DESENVOLVIMENTO}

Para determinação dos pontos de saída para o veículo espacial, foram consideradas uma órbita inicial e outra final, ambas circulares e discretizadas de $10 \mathrm{em}$ 10 graus, gerando um total de 36 pontos, conforme demonstrado na Figura 2.

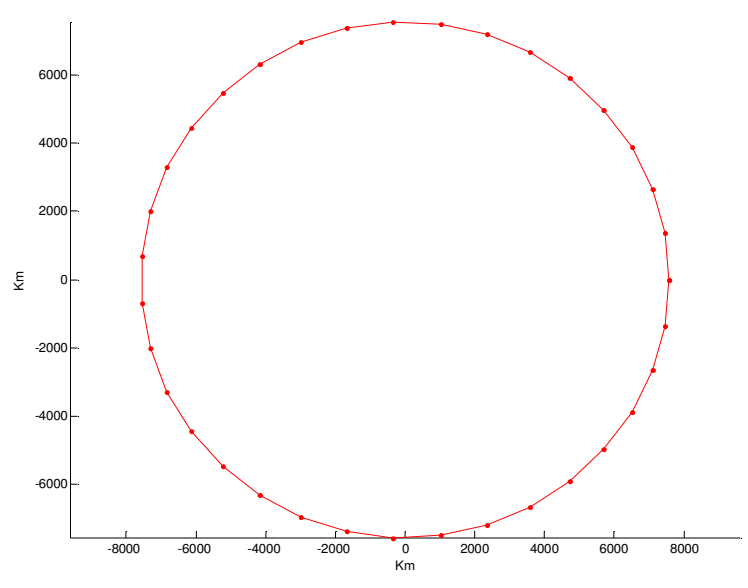

Figura 2 - Órbita inicial com os 36 possíveis pontos de saída.

A partir dos 36 pontos gerados dentro das órbitas, foram escolhidos quatro pontos para a saída do veículo espacial na órbita inicial, escolhidos de maneira uniforme ao longo da órbita, e um único ponto de chegada na órbita final.

\section{RESULTADOS}

Foram criados algoritmos para a transferência de Lambert e para o método de integração Runge-Kutta de $4^{\text {a }}$ ordem, aplicando nas equações da dinâmica do movimento. Para as trajetórias geradas a partir do integrador, considerando os 
propulsores ligados, foi obtido o resultado apresentado na Figura 3.

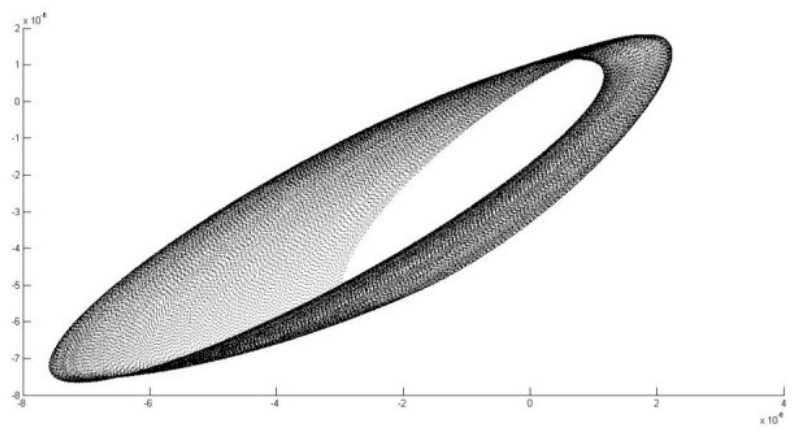

Figura 3 - Trajetória do veículo espacial com as órbitas aumentando com o tempo, de acordo com o impulso $\Delta \mathrm{V}$ aplicado pelo propulsor.

Para a simulação da dinâmica de dois pontos obtidos dos raios, foi obtida a corpos, variando o ângulo $\theta$ de $\theta_{\mathrm{i}}$ até $\circ \theta_{\mathrm{f}}$ seguinte trajetória de transferência, desejado, onde $\theta \in\left[\theta_{\mathrm{i}}, \theta_{\mathrm{f}}\right]$ e traçando os apresentado na Figura 4:

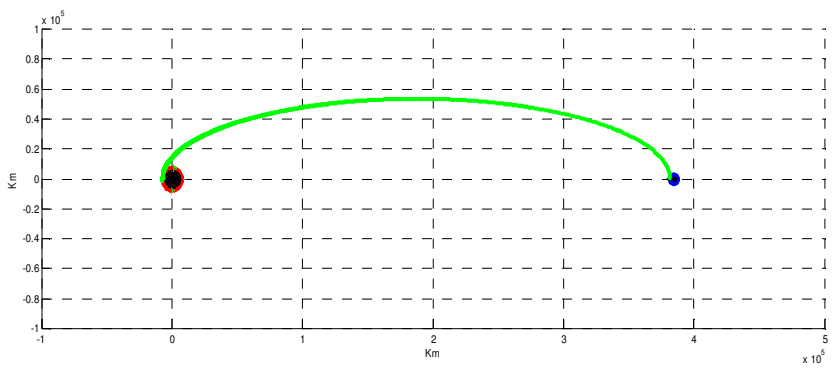

Figura 4 - Transferência Terra-Lua no sistema geocêntrico planar.

Para a simulação da dinâmica de três trajetórias, geradas a partir de uma órbita corpos, considerando 4 possíveis trajetórias, graduada de $90 \mathrm{em} 90^{\circ}$, foram plotados os um único ponto de chegada na órbita final e pontos obtidos a cada integração numérica, um mesmo tempo de transferência entre as conforme demonstrado na Figura 5:

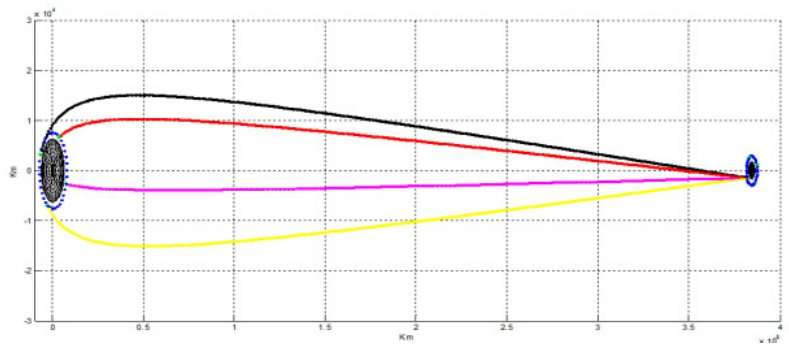

Figura 5 - Transferência Terra-Lua considerando 4 possíveis trajetórias. 
A Figura 6 demonstra uma corpo em cada instante e outra simulação comparação entre uma simulação utilizando utilizando uma dinâmica de dois corpos, por integração numérica para obter a posição do meio das equações kleperianas.

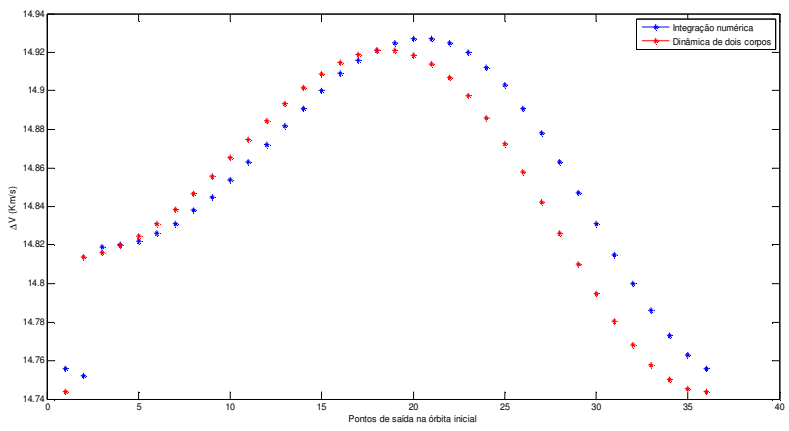

Figura 6 - Comparação entre a dinâmica de dois corpos e outra dinâmica utilizando integração numérica para obter a magnitude do impulso total necessário.

A partir desse gráfico, é possível identificar a posição angular de cada um dos possíveis pontos para saída do veículo espacial e a posição onde a magnitude do impulso para sair da Terra e entrar na órbita da Lua é mínima.

\section{CONCLUSÃO}

Foram desenvolvidos algoritmos para calcular e simular a trajetória de uma transferência realizada por um veículo espacial da Terra à Lua, considerando uma dinâmica de dois corpos e outra utilizando integração numérica.

A partir dessa etapa, serão implementados os algoritmos genéticos em populações de $\Delta \mathrm{t}$, para encontrar o melhor momento em que é obtido o menor impulso $\Delta \mathrm{V}$ para realizar a manobra de transferência, considerando o percurso Terra-Lua.

\section{AGRADECIMENTOS}

A Deus; à FAPESP, pelo apoio financeiro recebido, por intermédio da bolsa de Iniciação Científica; e aos orientadores,
Dr. Denilson Paulo Souza dos Santos e Dr. Antonio Fernando Bertachini de Almeida Prado, pelo conhecimento transmitido.

\section{REFERÊNCIAS}

CURTIS, H. D. Orbital Mechanics for Engineering Students. 2. ed. Amsterdam: Elsevier, 2010.

KIUSALAAS, J. Numerical Methods in Engineering with Python. Cambridge: Cambridge University Press, 2005.

LINDEN, R. Algoritmos Genéticos: Uma importante ferramenta da Inteligência Computacional. 2. ed. Rio de Janeiro: Brasport, 2008.

PRADO, A. F. B. A. Escola de Verão de Dinâmica Orbital e Planetologia, n. 16. Guaratinguetá, SP: UNESP, 2012. (Apostila).

SANTOS, D. P. S. Otimização de trajetórias espaciais com propulsão elétrica solar e manobras gravitacionalmente assistidas. 2009. Tese (Doutorado em Mecânica Espacial e Controle) Instituto Nacional de Pesquisas Espaciais, São José dos Campos, 2009. 